\title{
Motivos de consulta em Medicina Geral e Familiar no distrito de Coimbra no ano de 2010
}

Diogo Barreiro,* Luiz Miguel Santiago**

\section{RESUMO}

Objetivos: Caracterizar os motivos de consulta classificados pelos médicos de família.

Tipo de estudo: Descritivo e transversal.

Local: Distrito de Coimbra.

População: Utentes dos Centros de Saúde do distrito de Coimbra em 2010.

Métodos: Foram estudados 453.388 motivos de consulta, codificados utilizando a segunda edição da International Classification for Primary Care (ICPC-2), no distrito de Coimbra durante o ano de 2010 (dados fornecidos pela Administração Regional de Saúde do Centro). Foi utilizada estatística descritiva utilizando o programa PAWS vs 19.

Resultados: Os motivos de consulta mais frequentemente registados pelos médicos no distrito de Coimbra, no ano de 2010, foram, por ordem decrescente, dos capítulos «geral e inespecífico» $(39,8 \%)$, «sistema músculo-esquelético» (9,9\%) e «aparelho circulatório» (8,7\%). Por componentes, os mais registados, por ordem decrescente, foram «sinais e sintomas» (30,8\%), «procedimentos diagnósticos e preventivos» $(20,0 \%)$ e «resultados de exames» (19,3\%). Os sinais e sintomas mais frequentes foram «tosse» $(5,6 \%)$, «sinais/sintomas da região lombar» $(4,2 \%)$, «sinais/sintomas da garganta» $(3,5 \%)$ e «febre» $(3,5 \%)$. Conclusões: Os problemas gerais e inespecíficos foram os motivos de consulta mais frequentes na população em estudo.

Palavras-chave: Cuidados de Saúde Primários; Medicina Geral e Familiar; Consulta.

\section{INTRODUÇÃO}

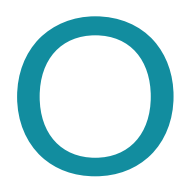

s Cuidados de Saúde Primários (CSP) e em particular a Medicina Geral e Familiar (MGF) são a porta de entrada no Serviço Nacional de Saúde e o que acontece neles deve ser estudado. Há pouco conhecimento sobre os motivos que levam as pessoas a procurar o seu médico de família (MF), o que pode conduzir a uma má abordagem e compreensão dos conceitos de prevenção, promoção e risco. ${ }^{1} \mathrm{Em}$ muitos países há falta de informação sobre os motivos que levam os utentes a dirigir-se aos CSP e em Portugal verifica-se o mesmo desconhecimento. Os registos informáticos são fundamentais em qualquer sistema de saúde ${ }^{2}$ e em MGF podem ser utilizados não só para avaliar o trabalho dos clíni-

* Mestre em Medicina pela Faculdade de Medicina da Universidade de Coimbra Centro Hospitalar e Universitário de Coimbra

**Consultor, Assistente Graduado Sénior de Medicina Geral e Familiar

ACES Baixo Mondego, ARS do Centro IP cos, como também para estudo de um extenso conjunto de informação disponível e de fácil acesso para investigação, fornecendo importantes dados epidemiológicos. O estudo do motivo de consulta e expetativas do doente permite o desenvolvimento da Saúde Pública e de programas de prevenção e o fornecimento adequado de cuidados diretos ao utente., ${ }^{2,3}$

O motivo de consulta é definido como «um termo acordado que transmite as razões pelas quais um paciente entra no sistema de saúde e representa o motivo desta pessoa». ${ }^{4}$ Os motivos pelos quais os pacientes procuram o seu MF podem ser extremamente variáveis, passando por pedido de procedimentos administrativos, de rastreio, de seguimento de doenças crónicas e de avaliação de sinais e sintomas, entre outros. ${ }^{4,5,6}$

Assim, o conhecimento da distribuição da frequência dos motivos de consulta poderá ter impacto na dinâmica da consulta e na rapidez e eficiência da atuação médica, em particular em MGF, quando são utili- 
zados instrumentos normalizados para conhecimento do que se pretende medir. ${ }^{7}$

A International Classification for Primary Care (ICPC) foi publicada em 1987 pelo Comité de Classificação Internacional daWONCA (World Organization of Family Physicians). A versão atual (ICPC-2) resulta da revisão da primeira versão (ICPC-1). ${ }^{5}$ A ICPC baseia-se numa estrutura simples bi-axial: 17 capítulos num dos eixos, cada um com um código alfa, e, no outro eixo, 7 componentes com rubricas numeradas com códigos de dois dígitos. No fim de cada secção e subsecção, podem encontrar-se rubricas residuais, cuja descrição inclui a palavra «outros» ou «não especificado de outra forma». A ICPC-2 permite a classificação padronizada dos motivos de consulta, podendo ser utilizada juntamente com o método de Weed. Assim, a ICPC-2 reflete elementos essenciais de cada encontro entre médico e utente, permitindo a anotação e posterior análise de vários dados decorrentes do encontro. Por um lado, a ICPC-2 é uma ferramenta útil e adequada na prática clínica, sendo proveitosa para o conhecimento psicossocial e posterior avaliação diagnóstica, tendo sido aceite pela Organização Mundial de Saúde como a melhor ferramenta de classificação nos CSP.; ${ }^{6-811}$ No entanto, apesar de ser uma ferramenta útil para a codificação, é pouco adequada para a prática clinica diária não protegida, caracterizada por grande pressão assistencial, pelo que classificar usando a ICPC é adequado sobretudo em ambientes protegidos: investigação, internatos e ensino, entre outros. ${ }^{12-13}$

Este estudo tem como objetivo verificar quais os motivos de consulta registados pelos MF do distrito de Coimbra, no ano de 2010, utilizando a ICPC-2, avaliando a frequência por capítulo e pelos diferentes componentes do capítulo.

\section{MATERIAIS E MÉTODOS}

Foi realizado um estudo observacional, transversal contabilizando os motivos de consulta codificados utilizando a ICPC-2 pelos MF do distrito de Coimbra durante o ano de 2010. Os ficheiros informáticos foram disponibilizados, em formato Excel®, pela Administração Regional de Saúde do Centro, após pedido da respetiva autorização, mediante aprovação do protocolo pela Comissão de Ética da Faculdade de Medicina da Universidade de Coimbra. Os centros de saúde estuda- dos utilizam a aplicação de registos médicos electróni$\cos \mathrm{SAM}$.

$\mathrm{O}$ distrito de Coimbra tem uma área de $3.947 \mathrm{~km}^{2} \mathrm{e}$ uma população residente de 429.714 habitantes (Instituto Nacional de Estatística, 2009), integrando $17 \mathrm{mu}$ nicípios (Arganil, Cantanhede, Coimbra, Condeixa-aNova, Figueira da Foz, Góis, Lousã, Mira, Miranda do Corvo, Montemor-o-Velho, Oliveira do Hospital, Pampilhosa da Serra, Penacova, Penela, Soure, Tábua e Vila Nova de Poiares). O estudo decorreu sobre uma amostra de conveniência dos MF de 16 CS localizados em 12 destes 17 municípios. Não foram incluídos os utentes dos CS dos municípios de Cantanhede, Figueira da Foz, Lousã, Soure e Penela devido à não disponibilização atempada dos dados.

Foi utilizada estatística descritiva e utilizado o programa PAWS vs 19.

\section{RESULTADOS}

Foram estudados 453.388 motivos de consulta codificados nos 16 CS do distrito de Coimbra estudados, resultantes de 945.348 encontros no ano 2010, correspondendo a 0,48 motivos por encontro.

No quadro I estão representados os motivos de consulta estudados, segundo o capítulo e o componente da ICPC-2. Os motivos de consulta mais frequentemente codificados pertenciam ao capítulo A «geral e inespecífico» (39,8\%). A estes seguiram-se os motivos do capítulo L «sistema músculo-esquelético» (9,9\%), do capítulo K «aparelho circulatório» (8,7\%) e do capítulo W «gravidez e planeamento familiar» $(7,8 \%)$.

Mais de um terço dos motivos de consulta $(30,8 \%)$ foram codificados no componente «sinais e sintomas» (Quadro I). Seguiram-se os motivos dos componentes «procedimentos diagnósticos e preventivos» $(20,0 \%)$, «resultados de exames» $(19,3 \%)$ e «medicações, tratamentos e procedimentos terapêuticos» $(16,6 \%)$.

Globalmente, o motivo de consulta mais frequente foi «resultados de análises/procedimentos» do capítulo «geral e inespecífico» $(13,2 \%)$, seguido de «consulta de seguimento não especificada» do mesmo capítulo $(4,6 \%)$ e de «exame médico/avaliação de saúde/parcial» do «gravidez e planeamento familiar» $(4,4 \%)$ (Quadro II).

Dos motivos de consulta do capítulo «geral e inespecífico», 30,0\% corresponderam ao componente «re- 


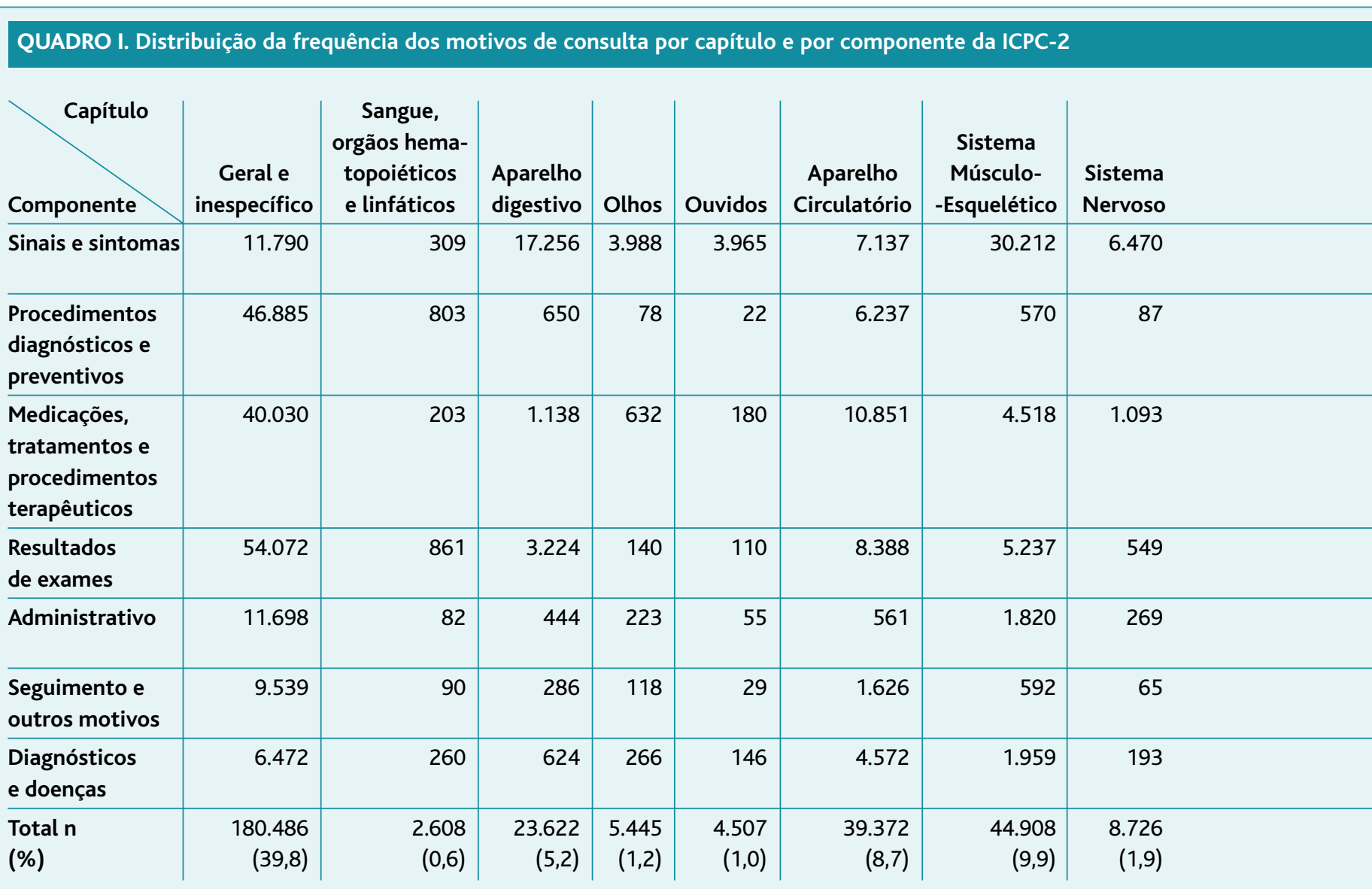

sultados de exames» e 26,0\% a "procedimentos diagnósticos e preventivos». «Sinais e sintomas» foram responsáveis por $6,5 \%$ dos motivos de consulta deste capítulo, sendo, destes, «febre» $(40,5 \%)$, «debilidade/cansaço geral» $(26,4 \%)$ e «sentir-se doente» $(7,0 \%)$ as codificações mais utilizadas.

O capítulo «aparelho circulatório» foi responsável por $8,7 \%$ dos motivos de consulta, sendo, destes, $27,6 \%$ para o componente «medicações, tratamentos e procedimentos terapêuticos», $21,3 \%$ para «resultados de exames» e $18,1 \%$ por «sinais e sintomas» $(28,2 \%$ dos quais «outros sinais/sintomas», $13,3 \%$ «fatores de risco de doença cardiovascular», $12,3 \%$ «tornozelos inchados/edema» e 10,0\% «palpitações/perceção dos batimentos cardíacos»).

$\mathrm{O}$ «sistema músculo-esquelético» correspondeu a $9,9 \%$ dos motivos de consulta, sendo, destes, $67,3 \%$ do componente «sinais e sintomas», $11,7 \%$ de «resultados de exames» e 10,1\% de «medicações, tratamentos e procedimentos terapêuticos». Dos sinais e sintomas deste sistema apresentados como motivo de consulta, 19,6\% eram da região lombar e 11,3\% do joelho.

Pertenciam ao capítulo «gravidez e planeamento familiar» $7,8 \%$ dos motivos de consulta estudados. Destes, $67,3 \%$ eram do componente «procedimentos diagnósticos e preventivos» e 21,8\% de «diagnóstico e doenças». No componente «sinais e sintomas» (apenas 2,7\% dos motivos deste capítulo) foram mais frequentemente assinalados «contraceção oral» $(27,7 \%)$, «questões sobre a gravidez» $(22,1 \%)$ e «medo de estar grávida» (14,5\%).

Considerando os capítulos em conjunto, o componente «sinais e sintomas» foi o mais codificado, correspondendo a um total de 139.748 motivos de consulta 


\begin{tabular}{|c|c|c|c|c|c|c|c|c|c|}
\hline Psicológico & $\begin{array}{c}\text { Aparelho } \\
\text { Respiratório }\end{array}$ & Pele & $\begin{array}{l}\text { Endócrino, } \\
\text { Metabólico } \\
\text { e Nutricional }\end{array}$ & $\begin{array}{c}\text { Aparelho } \\
\text { Urinário }\end{array}$ & $\begin{array}{l}\text { Gravidez e } \\
\text { Planeamento } \\
\text { Familiar }\end{array}$ & $\begin{array}{l}\text { Aparelho } \\
\text { Genital } \\
\text { Feminino }\end{array}$ & $\begin{array}{l}\text { Aparelho } \\
\text { Genital } \\
\text { Masculino }\end{array}$ & $\begin{array}{c}\text { Problemas } \\
\text { Sociais }\end{array}$ & $\begin{array}{c}\text { Total n } \\
\text { (\%) }\end{array}$ \\
\hline 9.867 & 23.194 & 10.793 & 1.450 & 5.615 & 964 & 4.349 & 791 & 1.626 & $\begin{array}{r}139.776 \\
(30,8)\end{array}$ \\
\hline 302 & 786 & 81 & 8.130 & 347 & 23.857 & 1.386 & 267 & 9 & $\begin{array}{r}90.497 \\
(20,0)\end{array}$ \\
\hline 6.047 & 2.016 & 537 & 5.668 & 291 & 977 & 305 & 615 & 9 & $\begin{array}{r}75.110 \\
(16,6)\end{array}$ \\
\hline 503 & 1.349 & 373 & 6.045 & 1.971 & 1.297 & 3.008 & 598 & 3 & $\begin{array}{r}87.728 \\
(19,3)\end{array}$ \\
\hline 469 & 370 & 182 & 188 & 146 & 308 & 286 & 72 & 111 & $\begin{array}{r}17.284 \\
(3,8)\end{array}$ \\
\hline 227 & 185 & 78 & 1.344 & 160 & 308 & 202 & 80 & 10 & $\begin{array}{r}14.939 \\
(3,3)\end{array}$ \\
\hline 1.109 & 524 & 824 & 2.688 & 262 & 7.743 & 295 & 117 & 0 & $\begin{array}{r}28.054 \\
\quad(6,2)\end{array}$ \\
\hline $\begin{array}{r}18.524 \\
(4,1)\end{array}$ & $\begin{array}{r}28.424 \\
(6,3)\end{array}$ & $\begin{array}{r}12.868 \\
(2,8)\end{array}$ & $\begin{array}{r}25.513 \\
(5,6)\end{array}$ & $\begin{array}{r}8.792 \\
(1,9)\end{array}$ & $\begin{array}{r}35.454 \\
(7,8)\end{array}$ & $\begin{array}{r}9.831 \\
(2,2)\end{array}$ & $\begin{array}{r}2.540 \\
(0,6)\end{array}$ & $\begin{array}{r}1.768 \\
(0,4)\end{array}$ & $\begin{array}{r}453.388 \\
(100)\end{array}$ \\
\hline
\end{tabular}

$(30,8 \%)$. De entre estes (Quadro III), os mais frequentemente codificados foram «tosse» $(5,6 \%)$, «sinais/sintomas da região lombar» $(4,2 \%)$, «sinais/sintomas da garganta» (3,5\%) e "febre" (3,5\%).

\section{DISCUSSÃO}

Este estudo, debruçando-se sobre codificações, é o primeiro a fornecer informação detalhada sobre os motivos que, na ótica do paciente, levam a população do distrito de Coimbra à consulta com o MF.

Assumiu-se que os MF do distrito de Coimbra codificavam regularmente na sua consulta. No entanto, verificam-se apenas 0,48 motivos por encontro, sugerindo que esta prática não está, de facto, implantada. Pode-se incentivar a codificação investindo na consciencialização de que através da codificação se podem retirar dados importantes para estudos de investigação.
A diversidade dos capítulos encontrados nos motivos de consulta está de acordo com o facto de a MGF ser a porta de entrada no Sistema Nacional de Saúde. ${ }^{7,12,13,19,20}$

Quase $40 \%$ dos motivos de consulta codificados inserem-se no capítulo "geral e inespecífico», sendo cerca de um terço do componente «resultados de exames». A importância dos diferentes componentes varia entre capítulos. O componente «sinais e sintomas» foi globalmente o mais frequentemente codificado $(30,8 \%$ de todos os motivos de consulta), assim como na maioria dos vários capítulos, à exceção dos "geral e inespecífico», "sangue, órgãos hematopoiéticos e linfáticos», «aparelho circulatório» e «gravidez e planeamento familiar». Estes resultados sugerem que os utentes procuram frequentemente os cuidados primários de saúde por sinais e sintomas e não só por padecerem de 


\begin{tabular}{|c|c|c|}
\hline Motivo de Consulta & $\mathbf{n}$ & $\begin{array}{l}\% \text { do total de motivos } \\
\text { de consulta }(n=453.388)\end{array}$ \\
\hline $\begin{array}{l}\text { Geral e inespecífico - Resultados } \\
\text { das análises/Procedimentos }\end{array}$ & 60.030 & 13,2 \\
\hline $\begin{array}{l}\text { Geral e inespecífico - Consulta } \\
\text { de seguimento não especificada }\end{array}$ & 20.711 & 4,6 \\
\hline $\begin{array}{l}\text { Gravidez e Planeamento Familiar } \\
\text { - Exame médico/Avaliação de } \\
\text { saúde/parcial }\end{array}$ & 19.846 & 4,4 \\
\hline $\begin{array}{l}\text { Geral e inespecífico - Medicação/ } \\
\text { /Prescrição/Pedido/Renovação/ } \\
\text { /Injecção }\end{array}$ & 12.280 & 2,7 \\
\hline $\begin{array}{l}\text { Geral e inespecífico - Exame } \\
\text { médico/Avaliação de saúde/completo }\end{array}$ & 10.626 & 2,3 \\
\hline Aparelho Respiratório - Tosse & 7.811 & 1,7 \\
\hline $\begin{array}{l}\text { Geral e inespecífico - Exame médico/ } \\
\text { /Avaliação de saúde/parcial }\end{array}$ & 7.448 & 1,6 \\
\hline $\begin{array}{l}\text { Geral e inespecífico - Resultados de } \\
\text { outro prestador - exame/análises/ } \\
\text { /registos/carta }\end{array}$ & 7.032 & 1,5 \\
\hline $\begin{array}{l}\text { Sistema Músculo-Esquelético - Sinais/ } \\
\text { /sintomas da região lombar }\end{array}$ & 5.902 & 1,3 \\
\hline $\begin{array}{l}\text { Aparelho Respiratório - Sinais/ } \\
\text { /sintomas da garganta }\end{array}$ & 4.869 & 1,1 \\
\hline Geral e inespecífico - Febre & 4.777 & 1,1 \\
\hline $\begin{array}{l}\text { Aparelho Circulatório - Resultados } \\
\text { das análises/procedimentos }\end{array}$ & 4.634 & 1,0 \\
\hline Gravidez & 4.501 & 1,0 \\
\hline $\begin{array}{l}\text { Aparelho Circulatório - Medicação- } \\
\text {-prescrição/pedido/renovação/injeção }\end{array}$ & 4.453 & 1,0 \\
\hline $\begin{array}{l}\text { Aparelho Circulatório - Consulta de } \\
\text { seguimento não especificada }\end{array}$ & 4.234 & 0,9 \\
\hline
\end{tabular}

nital feminino», «aparelho genital masculino» e "problemas sociais».

No estudo publicado em 1984, Lamberts et al. ${ }^{15}$ chegaram à conclusão de que a população holandesa recorria mais frequente à consulta de MGF por motivos do «sistema músculo-esquelético», dos aparelhos circulatório e respiratório e, apenas em quarto lugar, por motivos de tipo geral e inespecífico, revelando alguma sobreposição com os capítulos mais comuns no presente estudo. Em relação aos sinais e sintomas mais frequentes no estudo holandês, a grande maioria corresponde aos encontrados no nosso estudo, à exceção da contraceção que representou naquele $2,4 \%$ das codificações. É ainda de realçar a inclusão nas mais frequentemente utilizadas de 2 codificações do capítulo «problemas sociais» (problema conjugal e problema ocupacional), sendo este um capítulo que em Coimbra é pouco usado.

Em 1995, José Guilherme Jordão, ${ }^{16}$ na sua tese de doutoramento «A medicina geral e familiar: caracterização prática e sua influência no ensino pré-graduado», descreve que os três motivos de consulta mais frequentemente codificados são dos mesmos capítulos que os encontrados o nosso estudo, mas por diferente ordem: «sistema músculo-esquelético» (16,3\%), «aparelho circulatório» $(14,1) \mathrm{e}$ «geral e inespecífico» (13,3\%). Por componentes, os dois mais usados foram, como no nosso estudo, «sinais e sintomas» (55\%) e «procedimentos diagnós-

uma doença bem definida, o que vai de encontro aos resultados de outros estudos. ${ }^{15}$

Os 30 sinais e sintomas mais frequentemente $(57,1 \%)$ apresentados como motivo de consulta por parte dos doentes distribuem-se heterogeneamente. Deste conjunto de sinais e sintomas mais frequentes estão excluídos 7 capítulos: «sangue, órgãos hematopoiéticos e linfáticos», «olhos», «endócrino, metabólico e nutricional», «gravidez e planeamento familiar», «aparelho ge- ticos e preventivos» (18\%), sendo «medicações, tratamentos e procedimentos terapêuticos» o terceiro $(13,2 \%)$ no estudo de Jordão (e quarto no nosso).

Os resultados do nosso estudo por capítulos e por componentes também foram semelhantes aos encontrados no trabalho de Gabriel Rodrigues, de 2010, ${ }^{17}$ realizado no CS de Cascais, mas diferem quanto aos motivos de consulta mais frequentes, que em Cascais foram, por ordem decrescente: «exame médico/avalia- 


\begin{tabular}{|l|c|c|c|}
\hline \multicolumn{3}{|l|}{ QUADRO III. Sinais e sintomas mais frequentes no conjunto dos capítulos. } \\
\hline Sinais e Sintomas & $\mathbf{n}$ & $\begin{array}{c}\text { \% do total de sinais e } \\
\text { sintomas (n=139.776) }\end{array}$ & $\begin{array}{c}\text { \% do total de motivos } \\
\text { de consulta (n=453.388) }\end{array}$ \\
\hline Tosse & 7.811 & 5,6 & 1,7 \\
\hline Sinais/sintomas da região lombar & 5.902 & 4,2 & 1,3 \\
\hline Sinais/sintomas da garganta & 4.869 & 3,5 & 1,1 \\
\hline Febre & 4.777 & 3,4 & 1,1 \\
\hline Sinais/sintomas do joelho & 3.416 & 2,4 & 0,8 \\
\hline Debilidade/cansaço geral & 3.113 & 2,2 & 0,7 \\
\hline Sensação de ansiedade/nervosismo/tensão & 3.050 & 2,2 & 0,7 \\
\hline Cefaleia & 2.964 & 2,1 & 0,7 \\
\hline Sinais/sintomas dos ombros & 2.556 & 1,8 & 0,6 \\
\hline Erupção cutânea localizada & 2.508 & 1,8 & 0,6 \\
\hline Sinais/sintomas da perna/coxa & 2.444 & 1,8 & 0,5 \\
\hline Perturbação do sono & 2.382 & 1,7 & 0,5 \\
\hline Espirro/congestão nasal & 2.382 & 1,7 & 0,5 \\
\hline Vertigens/tonturas & 2.361 & 1,7 & 0,5 \\
\hline Sinais/sintomas do pescoço & 2.145 & 1,5 & 0,5 \\
\hline Dores abdominais/cólicas, geral & 2.129 & 1,5 & 0,5 \\
\hline
\end{tabular}

ção de saúde/parcial - circulatório», «renovação de prescrição - geral e inespecífico» e «renovação de prescrição - circulatório» (enquanto no nosso estudo foram, também por ordem decrescente, «resultados de análises/procedimentos - geral e inespecífico», «consulta de seguimento não especificada - geral e inespecífico» e «exame médico/avaliação de saúde/parcial gravidez e planeamento familiar»).

No trabalho de Gustavo Gusso, em 2009, no Brasil, ${ }^{18}$ os motivos de consulta mais frequentes também foram os relativos ao capítulo "geral e inespecífico», seguindo-se o «sistema músculo-esquelético», «aparelho circulatório», «aparelho respiratório», «aparelho digestivo» e «gravidez e planeamento familiar». Estes dados também coincidem com os nossos nos três primeiros capítulos, diferindo nos seguintes. Quanto aos sinais e sintomas mais frequentes, verificou-se uma grande semelhança com os agora encontrados em Coimbra, à exceção de muitos motivos por «secreção vaginal», «contraceção oral» $\mathrm{e}$ "prurido». Os motivos de consulta mais frequentes foram «Medicina preventiva/manu- tenção da saúde», seguido de «medicação/prescrição/ /renovação/injeção - circulatório» e de "febre», também diferentes dos do nosso estudo.

Se, quando considerados por capítulos e componentes, os resultados do nosso não diferem muito dos de outros estudos, quando considerados os motivos de consulta globalmente mais frequentes, existem diferenças entre todos os estudos realizados. Tal pode refletir diferenças reais nos motivos de consulta (decorrentes de diferentes sistemas de organização de cuidados ou de diferentes realidades epidemiológicas), ou diferenças na prática da codificação em si.

Como limitações deste estudo podem apontar-se: 0 desconhecimento sobre a proporção de MF que codifica motivos de consulta na sua prática diária; o desconhecimento sobre se os MF codificam todos os motivos de consulta de cada encontro com o utente; a eventual dificuldade dos clínicos em interpretarem alguns termos e conceitos apresentados pelo utente; e a possível utilização incorreta da ICPC-2 (apesar da existência de livro explicativo e de cursos para os médicos, 
quer especialistas, quer internos), resultando em certa probabilidade de a codificação não ser homogénea. Foi utilizada esta metodologia porque se assumiu que muitos médicos fazem codificação na sua prática diária e que, existindo já nesta região uma experiência de utilização das ferramentas de codificação de, em média, quatro anos, está no momento de se iniciar estudos sobre esta prática. Os dados criados pela generalização da prática de codificação devem ser estudados, no mínimo com objetivos formativos. O estudo dos dados resultantes da prática clínica diária assume-se assim como uma mais-valia adicional ao estudo das codificações de médicos especialmente treinados em contexto de investigação.

Concluindo, os motivos de consulta mais frequentes no distrito de Coimbra, no ano de 2010, utilizando a ICPC-2, foram, por ordem decrescente, do capítulo «geral e inespecífico», do "sistema músculo-esquelético» e do «aparelho circulatório». Por componentes, os motivos de consulta mais registados foram, por ordem decrescente, «sinais e sintomas», «procedimentos diagnósticos e preventivos» e «resultados de exames». Os sinais e sintomas que mais frequentemente motivaram consultas foram «tosse», «sinais/sintomas da região lombar»e «sinais/sintomas da garganta».

Os autores consideram importante o investimento na formação dos MF e internos de especialidade quanto ao uso da ICPC-2, para minimizar os erros de codificação. Consideram ainda que existe vantagem na codificação sistemática dos motivos de consulta de todos os encontros entre utente e médico. Devido à atual situação económica do país teria interesse repetir o estudo com dados mais recentes para averiguar uma possível alteração na codificação no capítulo «problemas sociais».

\section{REFERÊNCIAS BIBLIOGRÁFICAS:}

1. Gervas J, Pérez Fernandez M. El fundamento cientifico de la función de filtro del médico general. Rev Bras Epidemiol 2005 jun; 8 (2): 205-18.

2. de Lusignan S, Teasdale S, Little D, Zapp J, Zuckerman A, Bates DW, et al. Comprehensive computerised primary care records are an essential component of any national health information strategy: report from an international consensus conference. Inform Prim Care 2004; 12 (4): 255-64.

3. Pigeot I, De Henauw S, Foraita R, Jahn I, Ahrens W. Primary prevention from the epidemiology perspective: three examples from the practice. BMC Med Res Methodol 2010 Feb 3; 10 (1): 10.

4. The Wonca International Classification Committee World Organization of National Colleges, Academies and Academic Associations of General Practitioners/Family Physicians (WONCA). International Classification of Primary Care (ICPC-2-R). 2nd ed. Oxford: Oxford University Press; 1998. p. 2.

5. WONCA. ICPC-2-R: International Classification of Primary Care. Oxford: Oxford University Press; 2005.

6. WONCA. ICPC-2: International Classification of Primary Care. Oxford: Oxford University Press; 1987.

7. Queiroz MJ. SOAP revisitado. Rev Port Clin Geral 2009 Mar-Abr; 25 (2): 221-7.

8. van der Horst F, Metsemakers J, Vissers F, Saenger G, de Geus C. The Reason-for-Encounter mode of the ICPC: reliable, adequate, and feasible. Scand J Prim Health Care 1989 Jun; 7 (2): 99-103.

9. Okkes IM, Oskam SK, Van Boven K, Lamberts H. EFP. Episodes of care in Dutch Family Practice. Epidemiological data based on the routine use of the International Classification of Primary Care (ICPC) in the Transition Project of the Academic Medical Center/University of Amsterdam (1985-2003). In: Okkes IM, Oskam SK, Lamberts H. ICPC in the Amsterdam Transition Project. CD-Rom. Amsterdam: Academic Medical Center/University of Amsterdam, Department of Family Medicine; 2005.

10. Sampaio MM, Coeli CM, de Miranda NN, Faerstein E, Werneck GL, Chor D, et al. Confiabilidade interobservador da Classificação Internacional de Cuidados Primários. Rev Saude Publica 2008 jun; 42 (3): 536-41.

11. Chmiel C, Bhend H, Senn O, Zoller M, Rosemann T; FIRE study-group The FIRE project: a milestone for research in primary care in Switzerland. Swiss Med Wkly 2011 Jan 28; 140: w13142.

12. Melo M. O uso da ICPC nos registos clínicos em Medicina Geral e Familiar. Rev Port Med Geral Fam 2012 Jul-Ago; 28 (4): 245-6.

13. Pinto D. Classificar motivos de consulta e procedimentos com a ICPC na prática clínica? Rev Port Med Geral Fam 2012 Jul-Ago; 28 (4): 247-8.

14. Ricketts TC, Johnson-Webb KD, Taylor P. Definitions of Rural: A Handbook for Health Policy Makers and Researchers. A Technical Issues Paper prepared for the Federal Office of Rural Health Policy Health Resources and Services Administration. 1998. Disponível em: http://www.shepscenter.unc.edu/rural/pubs/report/ruralit.pdf [acedido em 17/01/2013].

15. Lamberts H, Meads S, Wood M. Classification of reasons why persons seek primary care: pilot study of a new system. Public Health Rep 1984 Nov-Dec; 99 (6): 597-605.

16. Jordão JG. A medicina geral e familiar: caracterização prática e sua influência no ensino pré-graduado [tese de doutoramento]. Lisboa: Faculdade de Medicina da Universidade de Lisboa; 1995.

17. Rodrigues J. Gabriel. Porque consultam os utentes o seu Médico de Família? Rev Port Clin Geral 2000 Nov-Dez; 16 (6): 442-52.

18. Gusso GD. Diagnóstico de demanda em Florianópolis utilizando a Classificação Internacional de Atenção Primária: $2^{\mathrm{a}}$ edição (CIAP-2) [tese de doutoramento]. São Paulo: Faculdade de Medicina da Universidade de São Paulo; 2009.

19. Santiago LM. Anotações, codificações e registos na consulta de Medicina Geral e Familiar. Rev Port Clin Geral 2012 Jul-Ago; 28 (4): 313.

20. Pinto D, Corte-Real S. Codificação com a Classificação Internacional de Cuidados Primários (ICPC) por internos de Medicina Geral e Familiar. Rev Port Clin Geral 2010 Jul-Ago; 26 (4): 370-82. 


\section{CONFLITO DE INTERESSES}

Os autores declaram não ter nenhum conflito de interesses relativamente ao presente artigo.

\section{FONTES DE FINANCIAMENTO}

Não existiram fontes externas de financiamento para a realização deste estudo.

\section{ENDEREÇO PARA CORRESPONDÊNCIA}

Rua da Misericórdia, n. ${ }^{3} 37$

Urb. D. Inês - 3100-525 Pombal

e.mail: diogobarreiro@hotmail.com

Recebido em 18/02/2013

Aceite para publicação em 22/07/2013

Artigo escrito ao abrigo do novo acordo ortográfico.

\section{ABSTRACT}

\section{REASONS FOR CLINICAL ENCOUNTER IN GENERAL PRACTICE/FAMILY MEDICINE IN 2010 IN CENTRAL PORTUGAL}

Objectives: To characterize the reasons for consultation in primary care as coded by family physicians.

Study design: Descriptive, cross-sectional.

Setting: District of Coimbra, Portugal.

Participants: Patients visiting health centres in the Coimbra district in 2010.

Methods: This study examined 453,388 reasons for consultation recorded in primary care in the district of Coimbra during the year 2010. They were coded using the International Classification for Primary Care, second edition (ICPC-2). Descriptive statistics were obtained using PAWS v19 statistical software.

Results: The most frequent reasons for consultation recorded were "general and unspecified" (39.8\%), "musculoskeletal system" $(9,9 \%)$ and "circulatory system" (8.7\%). The most commonly recorded components were "signs and symptoms" $(30.8 \%)$, "diagnostic and preventive procedures" (20.0\%) and "test results" (19.3\%). The most frequent signs and symptoms symptoms recorded were "cough" (5.6\%), "signs and symptoms in the lumbar region" (4.2\%), "signs/symptoms of the throat" (3.5\%) and "fever" (3.5\%).

Conclusions: General and unspecified conditions were found to be the most common reason for consultation in this population.

Keywords: Primary Health Care; Family Practice; Office visit. 